Información Tecnológica

Vol. 25(2), 123-130 (2014)

doi: $10.4067 / S 0718-07642014000200014$

\title{
Planificadores para Tareas en Tiempo Real Concurrentes: Una Descripción Basada en Teoría de Conjuntos
}

\author{
Jorge S. Valdez-Martínez*, Pedro Guevara-López, Gustavo Delgado-Reyes y Jesús Audelo-González \\ Instituto Politécnico Nacional, Sección de Estudios de Posgrado e Investigación, ESIME CUL, \\ Av. Santa Ana No. 1000 Col. San. Francisco Culhuacán Del. Coyoacán, C.P. 04430 México D.F.-México \\ (e-mail: jsvaldezmtz@yahoo.com.mx, pguevara@ipn.mx, dengue_mgs4@hotmail.com, \\ audelo77@gmail.com)
}

${ }^{*}$ Autor a quien debe ser dirigida la correspondencia

Recibido May. 28, 2013; Aceptado Jul. 24, 2013; Versión final recibida Sep. 24, 2013

\begin{abstract}
Resumen
En este artículo se proponen definiciones sobre las restricciones temporales de las etapas de una tarea en tiempo real (TTR). Estas definiciones se construyeron utilizando teoría de conjuntos y funciones, sirviendo de base para formalizar el concepto de planificador de tareas como una función que mapea del conjunto de tiempos de arribo al conjunto de tiempos de inicio en un conjunto de TTR concurrentes. La función es válida para cualquier planificador de tareas ya que es descriptiva y no depende del algoritmo de planificación ni tampoco del tiempo de arribo de las tareas. Como ejemplo de aplicación se presenta un estudio sobre el planificador Rate Monotonic donde se define un caso específico con restricciones temporales. El conjunto de definiciones obtenido, permitirá realizar estudios de estabilidad, funcionalidad, optimización y factibilidad y comparación con otros planificadores.
\end{abstract}

Palabras clave: tiempo de arribo, teoría de conjuntos, tiempo de inicio, planificador, sistema en tiempo real.

\section{Concurrent real-time tasks schedulers: A description Based on Set Theory}

\begin{abstract}
In this paper definitions for time constraints of steps of a real-time task (RTT) are presented. These definitions were constructed using set theory and functions theory and provided the basis for formalizing the concept of scheduler of tasks as a function that maps from the arrival times set to the start times set on a set of concurrent RTT. This definition is valid for any scheduler and its description does not depend of the scheduling algorithm or of the arrival time of the tasks. As an application, a study on the Rate Monotonic scheduler in which a specific case with time constraints is presented. The set of definitions will allow performing studies of stability, functionality, optimization, feasibility and comparison with other schedulers.
\end{abstract}

Keywords: arrival time, set theory, start time, scheduler, real-time system. 


\section{INTRODUCCIÓN}

Un sistema operativo en tiempo real (SOTR) a diferencia de los sistemas operativos de tiempo compartido, da preferencia a los procesos del mundo físico sobre los procesos del usuario, asignando recursos por prioridades y no por demanda. La principal característica de estos sistemas es la de brindar soporte para el manejo de tareas en tiempo real (TTR) concurrentes, las cuales tienen restricciones temporales impuestas por el mundo físico y compiten para ser atendidas, demandando recursos del procesador del sistema de computo en diferentes intervalos de tiempos; en esta competencia por los recursos, es el planificador de tareas en tiempo real concurrentes (PTTRC) quien decide que tarea será atendida y en que intervalo. El concepto general de planificador está determinado como: "Un criterio predefinido que asigna recursos a los procesos en forma ordenada tal que cada tarea es ejecutada hasta completarse" (Medel et al., 2007).

Tratándose de sistemas en tiempo real (STR) los planificadores se han definido con diferentes enfoques; por ejemplo Liu y Layland (1973) lo definieron como "un conjunto de reglas que determinan que tarea va a ser ejecutada en un tiempo determinado". Burns y Wellings (2009) mencionaron que "es un algoritmo que asigna los recursos del procesador a diferentes tareas y en diferentes momentos". Pandelis (2013) consideró que la planificación busca incrementar el número de tareas concluidas de un número finito de tareas. Ha y Liu (1994) mencionaron que un algoritmo de planificación es un manejador de prioridades, lo que concuerda con Goossens et al. (2003) bajo la consideración de ser de ese tipo si y solo si satisface la condición que para un par de tareas $J_{i}$ y $J_{j}$, $J_{i}$ tiene mayor prioridad que $J_{j}$ en algún instante de tiempo, entonces $J_{i}$ siempre tendrá mayor prioridad que $J_{j}$. Xu y Parnas (1990) propusieron que cada proceso $p \in P$ consiste de una secuencia finita de segmentos $p[0], p[1], \ldots, p[n[p]]$, donde $p[0]$ es el primer segmento y $p[n[p]]$ es el último segmento en el proceso $p$. Para cada segmento $i$, se define al tiempo de liberación como $r[i]$, al plazo como $d[i]$, al tiempo de ejecución como $c[i]$, asumiéndose que $r[i], d[i]$ y $c[i]$ tienen valores enteros; entonces, un planificador de un conjunto de procesos $P$ es una función total $\pi: U \rightarrow[0, \infty)$.

En el trabajo de Baruah y Goossens (2004), se planteó que para cualquier conjunto de tareas $J$, un planificador $S$ es un mapeo del producto cartesiano de los números reales y el conjunto de tareas de $\{0,1\}$, de tal manera que: $S=\mathrm{R} x J \rightarrow\{0,1\}$; con $S(t, j)$ igual a uno si $S$ asigna al procesador una instancia $j$ al instante de tiempo $t$ y cero en otro caso Afrati et al. (2005) definieron al planificador como una función $\sigma: V$ $\rightarrow N \times\left\{M_{1}, M_{2}\right\}$, donde $V$ es un conjunto de tareas parcialmente ordenadas, siendo $M_{1}$ y $M_{2}$ dos procesadores idénticos, tal que $\sigma(i)=\left(t_{i}, M_{i}\right)$ donde $t_{i}$, es el tiempo en el cual la tarea $i \in V$ inicia su ejecución y $M_{i} \in\left\{M_{1}, M_{2}\right\}$ es el procesador en el cual la tarea $i$ es asignada. Buttazzo (2011); definió que "una planificación es una asignación de tareas al procesador donde cada tarea es ejecutada hasta completarse"; esto es: $\sigma=\mathbf{R}^{+} \rightarrow \mathbf{N}$ tal que $\forall t \in \mathbf{R}^{+} \exists t_{1}, t_{2}$ donde $t \in\left[t_{1}, t_{2}\right)$ y $\forall t^{\prime} \in\left[t_{1}, t_{2}\right) \sigma(t)=\sigma\left(t^{\prime}\right)$; Donde $\sigma(t)$ es definida como una función escalón con $\sigma(t)=k$, con $k>0$ la tarea $J_{k}$ es ejecutada en $t$, cuando $\sigma(t)=0$ el CPU está inactivo.

En el estado del arte presentado, las definiciones no están expresadas formalmente y en su mayoría consideran restricciones temporales básicas (tiempo de inicio, tiempo de ejecución y plazo) sin considerar las prioridades de las tareas en tiempo real concurrentes involucradas. Es por eso que a través del uso de funciones y teoría de conjuntos así como la inclusión de otras restricciones temporales de las instancias de las tareas en tiempo real y la consideración de que cada tarea concurrente en tiempo real es independiente de otra tarea concurrente, se propone presentar un conjunto de definiciones formales que ayudaran al análisis exhaustivo de las restricciones temporales de las tareas periódicas en tiempo real en su primera activación y en cada múltiplo del tiempo de muestreo. La razón por la cual este enfoque se basa en el modelo de tareas periódicas, es debido a que este modelo es la base de la teoría de planificación de tiempo real, pues permite representar adecuadamente el comportamiento temporal del mundo real y es posible realizar análisis teóricos (Brocal y Balbastre, 2013). Esto permitirá obtener un mayor uso de los recursos, mejor rendimiento de los procesos de control, ahorro de energía y puede permitir la adaptación a entornos dinámicos así como de situaciones de sobrecarga. Permitiendo obtener información adicional acerca del comportamiento del sistema, márgenes de planificación y variabilidad. Además se pueden obtener mejores resultados en el modelado de las instancias o de las tareas en tiempo real, incluso en el diseño de criterios para asignar tareas en tiempo real a sistemas computacionales en sistemas con tolerancia a fallos.

\section{DESARROLLO}

Formalmente se define a una tarea en tiempo real (TTR) como una entidad ejecutable de trabajo $J_{i}$ que al menos es caracterizada por un tiempo de arribo y una restricción temporal, lo que permite analizar no solo la primera instancia sino que también la $k$-ésima instancia de la tarea en tiempo real. En este sentido gran parte de los trabajos que conforman el estado del arte revisado para este artículo, sólo consideran un conjunto de parámetros estáticos en la descripción de las TTR, lo que para efectos de planificabilidad no es lo más adecuado. Otras tendencias de investigación mencionadas en el trabajo de Brocal y Balbastre 
(2013) giran en torno a aumentar la flexibilidad de los plazos y periodos y así modificar al modelo de tareas periódicas mediante la definición de relaciones de precedencia entre tareas, la consideración de la problemática derivada del acceso a recursos compartidos y en este trabajo en específico se retoma la idea de variar los periodos para encontrar el mínimo común múltiplo de los periodos de las tareas concurrentes mediante la propuesta de un híper-periodo. Aunque estas tendencias pueden resultar atractivas, se partirá del hecho de que cada tarea concurrente en tiempo real es independiente de otra tarea concurrente y que por consecuencia sus restricciones temporales son distintas entre sí, lo que permite considerar la inclusión de ciertas restricciones temporales en el modelado de las TTRC.

Para Buttazzo (2011), la instancia $j_{i, k}$ de una tarea en tiempo real está definida mínimamente con la siguiente triada de restricciones temporales $j_{i, k}=\left(l_{i, k}, c_{i, k}, d_{i, k}\right)$ con $i=(1, \ldots, p), k=(1, \ldots, n)$ para $i, k, n, p \in \mathbf{Z}^{+}$, donde $i$ es el índice de la tarea y $p$ es el número de tareas, $k$ es el índice de la instancia y $n$ es el número de instancias, donde el tiempo de arribo absoluto $l_{i, k} \in \mathrm{R}^{+}$, de una instancia $j_{i, k}$ de una TTR $J_{i}$ está definido como el tiempo en que la instancia pide atención al procesador en relación con el origen temporal de referencia. El tiempo de ejecución $c_{i, k} \in \mathrm{R}^{+}$, es el tiempo en que la instancia $j_{i, k}$ de una TTR $J_{i}$ concluye sus operaciones, sin considerar los desalojos de ésta en el procesador. $\mathrm{Y}$ el plazo máximo $d_{i, k} \in \mathrm{R}^{+}$, es el límite temporal superior en relación con el origen temporal de referencia, antes del cual la instancia $j_{i, k}$ de una TTR $J_{i}$ debe finalizar; cuando se trata de tareas periódicas, $d_{i, k}$ crece de manera monotónica tal que $d_{i, k}=d_{i, k-1}+T_{i}$ donde $T_{i}$ es el periodo de activación de la instancia $j_{i, k}$ de una TTR $J_{i}$.

El tiempo de respuesta del sistema en tiempo real depende en gran parte del comportamiento de los tiempos de ejecución $c_{i, k}$ de cada instancia $j_{i, k}$ de cada tarea en tiempo real $J_{i}$ y dicho comportamiento fluctúa debido a varios factores. Stappert y Altenbernd (2000) y Bernat et al. (203) consideraron que el comportamiento fluctuante de los tiempos de ejecución $c_{i, k}$ se deben a los factores computacionales como son el caching, pipeline, la búsqueda de la ruta de ejecución considerando a la exclusión mutua, número de lazos, predicciones y otras interacciones. Debido a estas razones, el análisis hecho para las restricciones temporales de la primera instancia $j_{i, l}$ de una TTR $J_{i}$ puede no tener sentido para cuando se trata de un algoritmo de planificación basado en prioridades, pues para la $k$-ésima instancia $j_{i, k}$ de una TTR $J_{i}$ puede tener una distribución de las restricciones temporales diferente a $j_{i, 1}$.

Por esta razón, en el trabajo de Guevara et al. (2011) se presentó un modelo ampliado el cual incluyó restricciones temporales dinámicas que dependen tanto del planificador de tareas en tiempo real así como del tiempo de ejecución $c_{i, k}$, de tal manera que la instancia $j_{i, k}$ de una TTR fue definida con la quíntupla $j_{i, k}=\left(l_{i, k}, s_{i, k}, c_{i, k}, f_{i, k}, d_{i, k}\right) i, k \in \mathbf{Z}^{+}$, donde el tiempo de inicio $s_{i, k} \in \mathbf{R}^{+}$de una instancia $j_{i, k}$ de una TTR $J_{i}$ fue definido como el instante en que la instancia es atendida por el procesador con relación al origen temporal de referencia. El tiempo de finalizado $f_{i, k} \in \mathbf{R}^{+}$de una instancia $j_{i, k}$ de una TTR $J_{i}$ fue definido como el instante en que la instancia es terminada por el procesador con relación al origen temporal de referencia. Cabe aclarar que $l_{i, k}, c_{i, k}, d_{i, k}, s_{i, k}, f_{i, k}, \mathrm{y} T_{i}$ están dados en unidades temporales [UT]. Adicionalmente, Guevara et al. (2011) analizaron la dinámica de los tiempos de ejecución de un algoritmo programado a través de un sistema en tiempo real STR, utilizando para ello el sistema operativo de tiempo real STR como lo es QNX, y concluyeron que para tareas en tiempo real concurrentes, no es suficiente considerar parámetros estáticos en la descripción de estas tareas, pues la propia dinámica de estos tiempos de ejecución define un parámetro con dinámica cambiante.

Es por ello y en base a lo anterior que en este artículo se propone agregar tres restricciones temporales más para describir a la instancia de la TTR mediante la óctupla $j_{i, k}=\left(l_{i, k}, s_{i, k}, c_{i, k}, f_{i, k}, o_{i, k}, r_{i, k}, h_{i, k}, d_{i, k}\right) i, k \in \mathbf{Z}^{+}$ siendo $o_{i, k} \in \mathbf{R}^{+}$el tiempo de operación, el cual es la diferencia existente entre el tiempo arribo $l_{i, k}$ y el tiempo de inicio $s_{i, k}$ de la instancia $j_{i, k}$ de una TTR $J_{i}$; esto es $o_{i, k}=s_{i, k}-l_{i, k} \forall i, k, p \in \mathbf{Z}^{+}$. El tiempo excedente es $r_{i, k} \in \mathbf{R}^{+}$, se considera como la diferencia existente entre el tiempo de finalizado $f_{i, k}$ y el plazo máximo absoluto $d_{i, k}$ de la instancia $j_{i, k}$ de una TTR $J_{i}$; esto es $r_{i, k}=f_{i, k}-d_{i, k}$, para que la instancia no pierda su plazo deberá cumplirse que $r_{i, k} \leq 0$, con $i, k, n, p \in \mathbf{Z}^{+}$. Adicionalmente se establece $h_{i, k} \in \mathbf{R}^{+}$como el tiempo de holgura, el cual se define como el valor negativo de $r_{i, k}$, esto es: $h_{i, k}=-r_{i, k}$. De igual manera $o_{i, k}, r_{i, k}$ y $h_{i, k}$ están dados en unidades temporales [UT]. Entonces para un conjunto de tareas en tiempo real concurrentes, estas restricciones temporales se pueden observar en la figura 1, en donde se aprecia que de acuerdo a dinámicas cambiantes de los tiempos de ejecución $c_{i, k}$, las dinámicas de las restricciones temporales siguientes $o_{i, k}, r_{i, k}, h_{i, k}$, serán por consecuencia de dinámicas cambiantes, lo que permite definir aún mejor a una TTR a través de este modelo ampliado, en el que se agregan estos tres parámetros que permiten definir de una mejor manera a las TTR, pero no definen a los criterios de planificabilidad o a los distintos tipos de planificadores, sin embargo como se prueba al final del trabajo, si estas restricciones son tomadas en consideración por el planificador, se obtiene una descripción más formal del proceso de planificación, como se observará en el ejercicio que se aborda en la sección de resultados del presente artículo. 


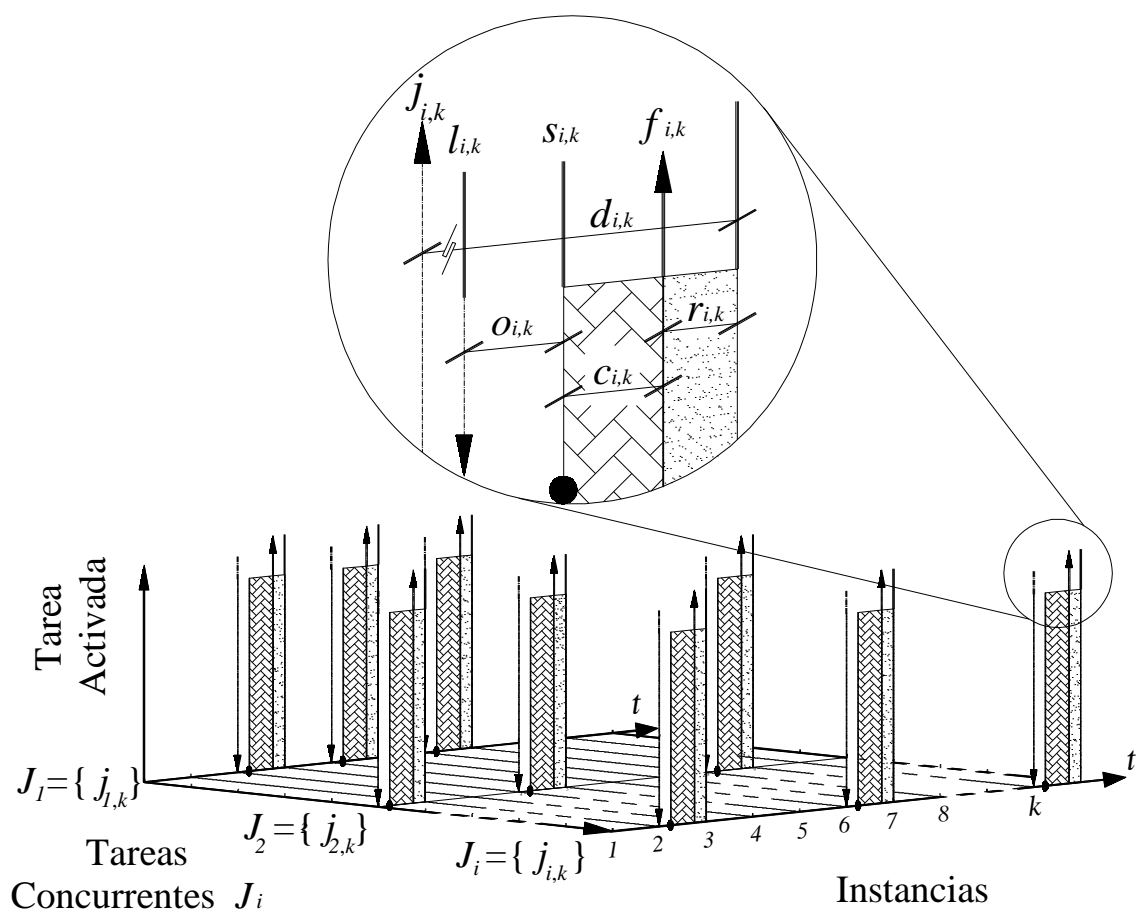

Fig. 1: Restricciones temporales de la instancia $j_{i, k}$ de la tarea en tiempo real concurrente $J_{i}$ : tiempo de arribo $l_{i, k}$, tiempo de inicio $s_{i, k}$, tiempo de ejecución $c_{i, k}$, tiempo de finalizado $f_{i, k}$, tiempo de operación $o_{i, k}$, tiempo excedente $r_{i, k}$, tiempo de holgura $h_{i, k}=-r_{i, k}$, y plazo $d_{i, k}$

Entonces teniendo en consideración la figura 1 y a (Guevara et al., 2012) se propone un conjunto de definiciones formales usando funciones y teoría de conjuntos como a continuación se establecen:

Definición 1 (Conjunto de Tareas en Tiempo Real Concurrentes). Un Conjunto de Tareas en Tiempo Real Concurrentes $\mathbf{J}=\left\{J_{1}, \ldots, J_{i}, \ldots, J_{p}\right\}$ con $i, p \in \mathbf{Z}^{+}$es aquel conjunto formado por al menos $p$ Tareas en Tiempo Real que compiten por $q$ recursos (procesadores, memoria, etc.) con la restricción de que $p>q$ con $p, q \in \mathbf{Z}^{+}$.

Definición 2 (Conjunto de Tiempos de Arribo). Sea un conjunto $\mathbf{J}$ de $p$ Tareas en Tiempo Real Concurrentes, cada tarea con $n$ instancias; $\mathbf{L}_{k}$ es el Conjunto de Tiempos de Arribo en el índice $k$ de las $j_{i, k}$ instancias, esto es $\mathbf{L}_{k}=\left\{l_{1, k}, \ldots, l_{i, k}, \ldots, l_{p, k}\right\}$ con $i, k, n, p \in \mathbf{Z}^{+}$.

Definición 3 (Conjunto de Tiempos de Inicio). Sea un conjunto $\mathbf{J}$ de $p$ Tareas en Tiempo Real Concurrentes, cada tarea con $n$ instancias; $\mathbf{S}_{k}$ es el conjunto de Tiempos de Inicio en el índice $k$ de las $j_{i, k}$ instancias, esto es $\mathbf{S}_{k}=\left\{s_{1, k}, \ldots, s_{i, k}, \ldots, s_{p, k}\right\}$ con $i, k, n, p \in \mathbf{Z}^{+}$.

Definición 4 (Conjunto de Tiempos de Ejecución). Sea un conjunto $\mathbf{J}$ de $p$ Tareas en Tiempo Real Concurrentes, cada tarea con $n$ instancias; $\mathbf{C}_{k}$ es el conjunto de Tiempos de Ejecución en el índice $k$ de las $j_{i, k}$ instancias, esto es $\mathbf{C}_{k}=\left\{c_{1, k}, \ldots, c_{i, k}, \ldots, c_{p, k}\right\}$ con $i, k, n, p \in \mathbf{Z}^{+}$.

Definición 5 (Conjunto de Tiempos de Finalizado). Sea un conjunto $\mathbf{J}$ de $p$ Tareas en Tiempo Real Concurrentes, cada tarea con $n$ instancias; $\mathbf{F}_{k}$ es el Conjunto de Tiempos de Finalizado en el índice $k$ de las $j_{i, k}$ instancias, esto es $\mathbf{F}_{k}=\left\{f_{1, k}, \ldots, f_{i, k}, \ldots, f_{p, k}\right\}$ con $i, k, n, p \in \mathbf{Z}^{+}$.

Definición 6 (Conjunto de Tiempos de Operación). Sea un conjunto $\mathbf{J}$ de $p$ Tareas en Tiempo Real Concurrentes, cada tarea con $n$ instancias; $\mathbf{O}_{\boldsymbol{k}}$ es el conjunto de Tiempos de Operación en índice $k$ de las $j_{i, k}$ instancias, esto es $\mathbf{O}_{k}=\left\{o_{1, k}, \ldots, o_{i, k}, \ldots, o_{p, k}\right\}$ con $i, k, n, p \in \mathbf{Z}^{+}$.

Definición 7 (Conjunto de Tiempos Excedentes). Sea un conjunto $\mathbf{J}$ de $p$ Tareas en Tiempo Real Concurrentes, cada tarea con $n$ instancias; $\mathbf{R}_{k}$ es el conjunto de Tiempos Excedentes en el índice $k$ de las $j_{i, k}$ instancias, esto es $\mathbf{R}_{k}=\left\{r_{1, k}, \ldots, r_{i, k}, \ldots, r_{p, k}\right\}$ con $i, k, n, p \in \mathbf{Z}^{+}$. 
Definición 8 (Conjunto de Tiempos de Holgura). Sea un conjunto $\mathbf{J}$ de $p$ Tareas en Tiempo Real Concurrentes, cada tarea con $n$ instancias; $\mathbf{H}_{k}$ es el conjunto de Tiempos de Holgura en el índice $k$ de las $j_{i, k}$ instancias, esto es $\mathbf{H}_{k}=\left\{h_{1, k}, \ldots, h_{i, k}, \ldots, h_{p, k}\right\}$ con $i, k, n, p \in \mathbf{Z}^{+}$.

Definición 9 (Conjunto de Plazos Máximos). Sea un conjunto J de $p$ Tareas en Tiempo Real Concurrentes, cada tarea con $n$ instancias; $\mathbf{L}_{k}$ es el Conjunto de Plazos Máximos en el índice $k$ de las $j_{i, k}$ instancias, esto es $\mathbf{D}_{k}=\left\{d_{1, k}, \ldots, d_{i, k}, \ldots, d_{p, k}\right\}$ con $i, k, n, p \in \mathbf{Z}^{+}$.

Considerando las definiciones obtenidas usando teoría de conjuntos de las restricciones temporales de las instancias de las tareas en tiempo real concurrentes, se llega a una definición formal del planificador mediante el uso de funciones:

Definición 10 (Planificador de Tareas en Tiempo Real Concurrentes). Todo planificador de tareas en tiempo real concurrentes (PTTRC) está definido como una función $\sigma\left(\mathbf{L}_{k}\right)$ que mapea del conjunto de tiempos de arribo $\mathbf{L}_{k}$ al conjunto de tiempos de inicio $\mathbf{S}_{k}$. Es decir $\sigma\left(\mathbf{L}_{k}\right): \mathbf{L}_{k} \rightarrow \mathbf{S}_{k}$, con $k \in \mathbf{Z}^{+}$.

Al ser el planificador una función $\sigma\left(\mathbf{L}_{k}\right)$ que mapea del conjunto de tiempos de arribo $\mathbf{L}_{k}$ al conjunto de tiempos de inicio $\mathbf{S}_{k}$ de tareas en tiempo real, este necesita manejar identificadores que le permitan decidir que tarea predominara una sobre otra, a este identificador se le llamara Prioridad de ejecución, el cual se define en este trabajo usando teoría de conjuntos y funciones como:

Definición 11 (Prioridad de Ejecución). La prioridad de ejecución $\alpha_{i, k}$ de toda instancia $j_{i, k} \in J_{i}$ es su nivel de preferencia para ejecutarse respecto a la instancia $j_{g, h} \in J_{u}$ con prioridad $\alpha_{g, h}$ tal que $\alpha_{i, k} \neq \alpha_{g, h}$ y $g, h, i, k, \alpha_{i, k}, \alpha_{i, k}$ $\in \mathbf{Z}^{+}$siempre que $j_{i, k}$ y $j_{g, h}$ se ejecuten en el intervalo $\left(l_{i, k}, d_{i, k}\right]$.

\section{RESULTADOS}

Para mostrar la utilidad de las definiciones anteriormente presentadas así como la definición de planificador de tareas en tiempo real concurrentes, se propone revisar un caso de la evolución temporal de un conjunto de TTR concurrentes $\mathbf{J}$ cuya asignación de prioridades fue hecha por el algoritmo de planificación RM (de las siglas en ingles de Rate Monotonic) el cual fue desarrollado por Liu y Layland (1973) y consiste en asignar mayor prioridad $\alpha_{i, k}$ a las tareas que tienen el menor periodo $\boldsymbol{T}_{\boldsymbol{i}}$ dentro del sistema. Para este ejemplo se presenta un conjunto de tareas periódicas concurrentes con las características mostradas en la tabla 1. En la tabla, $\boldsymbol{c}_{\mathbf{i}, k}$ y $\boldsymbol{T}_{\boldsymbol{i}}$ están expresados en unidades temporales [UT] y $\alpha_{i, k}$ no tiene unidades y se considera que $\alpha_{1, k} \ll \alpha_{3, k}$. Cuyos valores proporcionan una evolución temporal del conjunto tareas en tiempo real concurrentes mostrada en la figura 2.

Tabla 1: Características del conjunto de tareas concurrentes $\mathbf{J}=\left\{J_{1}, J_{2}, J_{3}\right\}$

\begin{tabular}{lccc}
\hline & $c_{i, k}[\mathrm{UT}]$ & $T_{i}[\mathrm{UT}]$ & $\alpha_{i, k}[\mathrm{UT}]$ \\
\hline Tarea $J_{1}$ & 32 & 80 & 1 \\
\hline Tarea $J_{2}$ & 5 & 40 & 2 \\
\hline Tarea $J_{3}$ & 4 & 16 & 3 \\
\hline
\end{tabular}

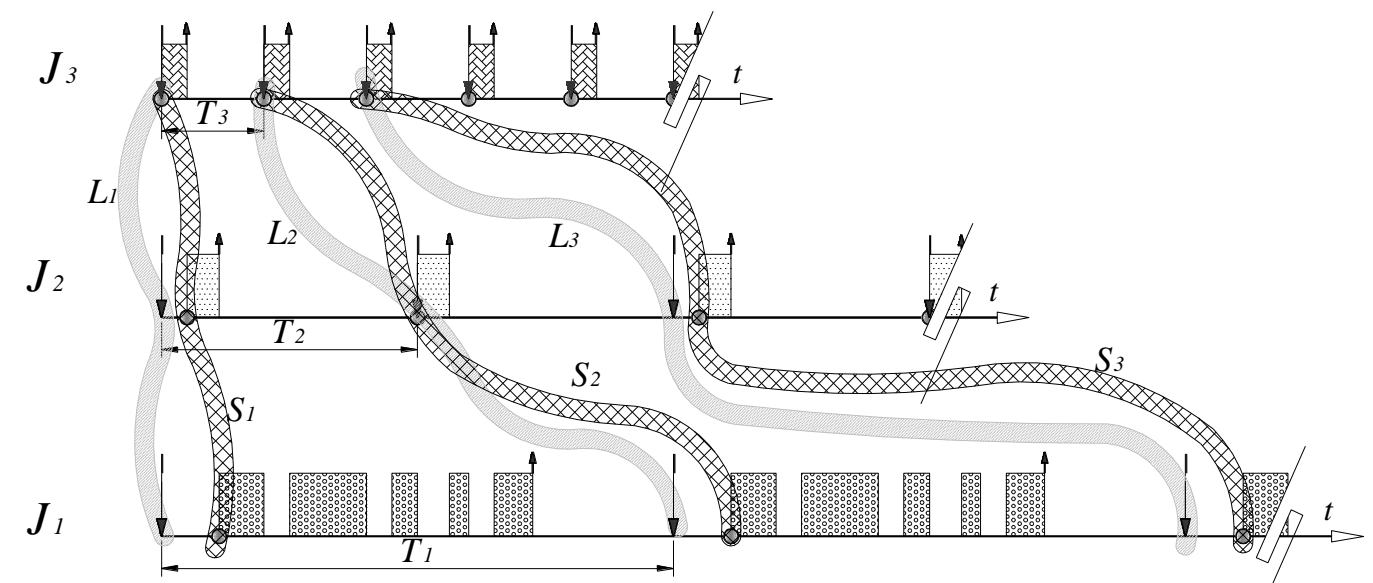

Fig. 2: Evolución temporal del conjunto de TTR concurrentes y los conjuntos de tiempos de arribo $\mathbf{L}_{k}$, el conjunto de tiempos de inicio $\boldsymbol{S}_{k}$ debido al efecto del planificador de tareas en tiempo real concurrentes $\sigma\left(\mathbf{L}_{k}\right)$. 
Tomando como referencia la figura 2, se obtienen los valores mostrados en la tabla 2 para el conjunto de restricciones temporales de las tareas en tiempo real concurrentes.

Tabla 2: Valores del conjunto de tareas concurrentes $\mathbf{J}=\left\{J_{l}, J_{2}, J_{3}\right\}$

\begin{tabular}{|c|c|c|c|c|c|c|c|c|c|}
\hline & & & & a) & Tarea $J_{1}$ & & & & \\
\hline & $l_{1, k}[\mathrm{UT}]$ & $s_{1, k}[\mathrm{UT}]$ & $c_{1, k,}[\mathrm{UT}]$ & $T_{1}[\mathrm{UT}]$ & $f_{1, k}[\mathrm{UT}]$ & $\alpha_{1, k}$ & $0_{1,}[\mathrm{UT}]$ & $r_{1, k}[\mathrm{UT}]$ & $U_{1, k}$ \\
\hline 1 & 0 & 9 & 32 & 80 & 58 & 1 & 9 & -22 & 0.400 \\
\hline 2 & 80 & 89 & 32 & 80 & 138 & 1 & 9 & -22 & 0.400 \\
\hline \multirow[t]{2}{*}{3} & 160 & 169 & 32 & 80 & 218 & 1 & 9 & -22 & 0.400 \\
\hline & \multicolumn{9}{|c|}{ a) Tarea $J_{2}$} \\
\hline$k$ & $I_{2, k}[\mathrm{UT}]$ & $s_{2, k}[\mathrm{UT}]$ & $c_{2, k}[\mathrm{UT}]$ & $T_{2}[\mathrm{UT}]$ & $f_{2, k}[\mathrm{UT}]$ & $\alpha_{2, k}$ & $\left.o_{2, k} \mathrm{UT}\right]$ & $r_{2, k}[\mathrm{UT}]$ & $U_{2, k}$ \\
\hline 1 & 0 & 4 & 5 & 40 & 9 & 2 & 4 & -31 & 0.125 \\
\hline 2 & 40 & 40 & 5 & 40 & 45 & 2 & 0 & -35 & 0.125 \\
\hline \multirow[t]{2}{*}{3} & 80 & 84 & 5 & 40 & 89 & 2 & 4 & -31 & 0.125 \\
\hline & \multicolumn{9}{|c|}{ b) Tarea $J_{3}$} \\
\hline$k$ & $l_{3, k}[\mathrm{UT}]$ & $s_{3, k}[\mathrm{UT}]$ & $c_{3, k}[\mathrm{UT}]$ & $T_{3}[\mathrm{UT}]$ & $f_{3, k}[\mathrm{UT}]$ & $\alpha_{3, k}$ & $o_{3, k}[\mathrm{UT}]$ & $r_{3, k}[\mathrm{UT}]$ & $U_{3, k}$ \\
\hline 1 & 0 & 0 & 4 & 16 & 4 & 3 & 0 & -12 & 0.250 \\
\hline 2 & 16 & 16 & 4 & 16 & 20 & 3 & 0 & -12 & 0.250 \\
\hline 3 & 32 & 32 & 4 & 16 & 36 & 3 & 0 & -12 & 0.250 \\
\hline
\end{tabular}

En donde $l_{i, k}, s_{i, k}, c_{i, k}, f_{i, k}, o_{i, k}, r_{i, k}, h_{i, k}, d_{i, k}$ están dados en unidades temporales [UT] y $U_{i, k}$ es la fracción de uso del tiempo del procesador en la ejecución del conjunto de tareas que para este ejemplo es constante debido a que son tareas periódicas, el cual está definido por Liu y Layland (1973):

$U_{k}=\sum_{i=1}^{p} \frac{C_{i, k}}{T_{i, k}}=0.775$

De la figura 2 y de la tabla 2 se obtienen los siguientes conjuntos. Aplicando el planificador $\sigma\left(\mathbf{L}_{k}\right)$ se tienen los mapeos entre conjuntos correspondientes:
$\mathbf{L}_{1}=\left\{l_{1,1}, l_{2,1}, l_{3,1}\right\}=\{0,0,0\}$,
$\sigma\left(\mathbf{L}_{1}\right): \mathbf{L}_{1} \rightarrow \mathbf{S}_{1}$;
$\mathbf{S}_{1}=\left\{s_{1,1}, S_{2,1}, S_{3,1}\right\}=\{9,4,0\} ;$
$\mathbf{L}_{2}=\left\{l_{1,2}, l_{2,2}, l_{3,2}\right\}=\{80,40,16\} ;$
$\sigma\left(\mathbf{L}_{2}\right): \mathbf{L}_{2} \rightarrow \mathbf{S}_{2}$
$\mathbf{S}_{2}=\left\{s_{1,2}, s_{2,2}, s_{3,2}\right\}=\{89,40,16\} ;$
$\mathbf{L}_{3}=\left\{l_{1,3}, l_{2,3}, l_{3,3}\right\}=\{160,80,32\} ;$
$\sigma\left(\mathbf{L}_{3}\right): \mathbf{L}_{3} \rightarrow \mathbf{S}_{3}$;
$\mathbf{S}_{3}=\left\{s_{1,3}, S_{2,3}, S_{3,3}\right\}=\{169,84,32\}$;
$\mathbf{C}_{1}=\left\{c_{1,1}, c_{2,1}, c_{3,1}\right\}=\{32,5,4\} ;$
$\mathbf{F}_{1}=\left\{f_{1,1}, f_{2,1} f_{3,1}\right\}=\{58,9,4\} ;$
$\mathbf{F}_{2}=\left\{f_{1,2}, f_{2,2} \cdot f_{3,2}\right\}=\{138,45,20\} ;$
$\mathbf{F}_{3}=\left\{f_{1,3}, f_{2,3}, f_{3,3}\right\}=\{218,89,36\} ;$
$\mathbf{D}_{1}=\left\{d_{1,1}, d_{2,1}, d_{3,1}\right\}=\{80,40,16\} ;$
$\mathbf{C}_{2}=\left\{c_{1,2}, c_{2,2}, c_{3,2}\right\}=\{32,5,4\}$;
$\mathbf{D}_{2}=\left\{d_{1,2}, d_{2,2}, d_{3,2}\right\}=\{160,80,32\}$;
$\mathbf{C}_{3}=\left\{c_{1,3}, c_{2,3}, c_{3,3}\right\}=\{32,5,4\}$;
$\mathbf{D}_{3}=\left\{d_{1,3}, d_{2,3}, d_{3,3}\right\}=\{240,120,48\}$;

Para los tiempos de operación $o_{i, k}$, los tiempos excedentes $r_{i, k}$ y los tiempos de holgura $h_{i, k}$ se tiene que:
$\mathbf{O}_{1}=\left\{o_{1,1}, o_{2,1}, o_{3,1}\right\}=\{9,4,0\} ;$
$\mathbf{R}_{1}=\left\{r_{1,1}, r_{2,1}, r_{3,1}\right\}=\{-22,-31,-12\} ;$
$\mathbf{H}_{1}=\left\{h_{1,1}, h_{2,1}, h_{3,1}\right\}=\{22,31,12\} ;$
$\mathbf{O}_{2}=\left\{o_{1,2}, o_{2,2}, o_{3,2}\right\}=\{9,0,0\}$
$\mathbf{R}_{2}=\left\{r_{1,2}, r_{2,2}, r_{3,2}\right\}=\{-22,-35,-12\}$;
$\mathbf{H}_{2}=\left\{h_{1,2}, h_{2,2}, h_{3,2}\right\}=\{22,35,12\}$;
$\mathbf{O}_{3}=\left\{o_{1,3}, o_{2,3}, o_{3,3}\right\}=\{9,4,0\}$
$\mathbf{R}_{3}=\left\{r_{1,3}, r_{2,3}, r_{3,3}\right\}=\{-22,-31,-12\}$;
$\mathbf{H}_{3}=\left\{h_{1,3}, h_{2,3}, h_{3,3}\right\}=\{22,31,12\}$;

\section{CONCLUSIONES}

Partiendo del hecho de que cada tarea concurrente en tiempo real es independiente de otra tarea concurrente, se pueden obtener las siguientes conclusiones sobre la descripción basada en teoría de conjuntos de los planificadores de tareas en tiempo real: 
1) El uso de la teoría de conjuntos así como de funciones es una herramienta que ayudó a la formalización de conceptos de las definiciones de las restricciones temporales de las tareas en tiempo real concurrentes: Conjunto de tiempos de arribo $l_{i, k}$, Conjunto de tiempos de inicio $s_{i, k}$, Conjunto de tiempos de ejecución $c_{i, k}$, Conjunto de tiempos de finalizado $f_{i, k}$, Conjunto de tiempos de operación $o_{i, k}$, Conjunto de tiempos excedentes $r_{i, k}$, Conjunto de tiempos de holgura $h_{i, k}$ y Conjunto de plazos $d_{i, k}$, así como la definición formal de planificador como una función que mapea del conjunto de tiempos de arribo al conjunto de tiempos de inicio, esto es $\sigma\left(\mathbf{L}_{k}\right): \mathbf{L}_{k} \rightarrow \mathbf{S}_{k}$, lo que permite tratar al concepto del Planificador para tareas concurrentes en tiempo real no como una descripción verbal informal sino como una definición reforzada usando teoría de conjuntos.

2) El conjunto de definiciones obtenido, permitirá realizar estudios de estabilidad, funcionalidad, optimización, factibilidad entre otros sobre los planificadores, así como una posible clasificación usando estas definiciones planteadas.

3) Los resultados obtenidos de la revisión de la evolución temporal de un conjunto de tareas en tiempo real concurrentes hecha por el algoritmo de planificación Rate Monotonic, pueden ser agrupados usando las definiciones propuestas en este documento.

4) Este conjunto de definiciones pueden ser usadas en otros algoritmos de planificación (FIFO, Deadline Monotonic (DM), Earliest Deadline First (EDF), Least Laxity First (LLF), Shortest Slack Time First (SSTF), etc.) para agrupar la evolución temporal del efecto de su planificación sobre un conjunto de tareas.

\section{REFERENCIAS}

Afrati F., E. Bampis, L. Finta y I. Milis, Scheduling trees with large communication delays on two identical processors. Journal of Scheduling ISSN: 1094-6136 (Impreso) 1099-1425 (En línea), 8(2), 179-190 (2005) http://link.springer.com/article/10.1007\%2Fs10951-005-6366-3 Acceso: 6 de Agosto (2013).

Baruah S. y J. Goossens, Handbook of Scheduling: Algorithms, Models, and Performance Analysis, 2-4. Chapman Hall/CRC Press, Bruselas, Bélgica (2004).

http://parts.ulb.ac.be//media/articles/baruahGoossens2003-3.pdf Acceso: 2 de Abril (2013).

Bernat G., A. Colin, S. M. Petters, PWCET: A tool for probabilistic Worst-Case Execution Time Analysis of Real-Time Systems, 1-18, Technical Report YCS-2003-353 of Department of Computer Science, University of York, Heslington York, UK (2003).

http://citeseerx.ist.psu.edu/viewdoc/download?doi=10.1.1.124.6696\&rep=rep1\&type=pdf Acceso: 6 de Agosto (2013).

Brocal V. y P. Balbastre, Selección del periodo para minimización del hiperperiodo. Revista Iberoamericana de Automatica e Informática Industrial, ISSN: 1697-7912 (Impreso), 10(2), 186-196 (2013). http://www.elsevierciencia.com/es/revista/revista-iberoamericana-automatica-e-informatica-industrial-

331/articulo/seleccion-del-periodo-minimizacion-del-hiperperiodo-90195914 Acceso: 21 de Septiembre (2013).

Burns A. y A. Wellings, Real-Time Systems and Programming Languages: : Ada, Real-Time Java and C/Real-Time POSIX, 4ª edición, 365-370, Editorial Addison Wesley Longmain, Reino Unido, Londres (2009).

Buttazzo G., Hard real-time computing systems: "Predictable scheduling, algorithms and applications", 3" edición, 24, Springer Science+Business Media, EUA, Nueva York (2011).

Goossens J., S. Funk y S. Baruah, Priority-driven scheduling of periodic task systems on multiprocessors, Real Time Systems, ISSN: 0922-6443 (Impreso), 1573-1383 (En línea), 25(2-3), 187-205 (2003). http://link.springer.com/article/10.1023/A\%3A1025120124771 Acceso: 2 de Abril (2013).

Guevara P., J. J. Medel y G. Delgado, Description of execution time dynamics for a set of concurrent realtime tasks, Revista Facultad de Ingeniería: Universidad de Antioquía, ISSN 0120-6230 (Impreso), (61), 123131 (2011). http://ingenieria.udea.edu.co/grupos/revista/revistas/nro061/Articulo\%2012.pdf. Acceso: 2 de Abril (2013).

Guevara P., O. Morales y J. Falcón, Concurrent real-time schedulers, a classification based on functions, Studies in Informatics and Control Journal, ISSN 1220-1766, (Impreso). 21(1), 27-32 (2012). http://sic.ici.ro/sic2012_1/art03.php Acceso: 2 de Abril (2013). 
Ha R. y J. Liu, Validating timing constrains in multiprocessor and distributed real-time systems, Proceedings of IEEE 14th International Conference on Distributed Computing Systems, 162-171, Poznan, Polonia, 21 al 24 de Junio (1994).

Liu C. y J. Layland, Scheduling algorithms for multiprogramming in a hard real-time environment, Journal of the ACM, ISSN: 0004-5411, (Impresa) 20(1), 46-61 (1973).

http://dl.acm.org/citation.cfm?id=321743\&CFID=351443004\&CFTOKEN=59003529. Acceso: 2 de Abril (2013).

Medel J. J., P. Guevara y D. Cruz, Temas selectos de sistemas en tiempo real, $1^{\text {era }}$ Edición, 50-54, Editorial Politécnico, México, Distrito Federal (2007).

Pandelis D. A note on preemptive scheduling of multiclass jobs with geometric service times and hard deadlines. Journal of scheduling ISSN: $1094-6136$ (Print) 1099-1425 (Online) 16(4), 395-403, (2013). http://link.springer.com/article/10.1007/s10951-012-0293-x Acceso: 6 de Agosto (2013).

Stappert F. y P. Altenbernd, Complete Worst-Case Execution Time Analysis of Straight-line Hard Real-Time Programs. Journal of Systems Architecture, ISSN: 1383-7621 (Impreso) 46(4), $339-355$ (2000). http://www.sciencedirect.com/science/article/pii/S1383762199000107 Acceso: 6 de Agosto (2013).

Xu J. y D. Parnas, Scheduling processes with release times, deadlines, precedence and exclusion relations, IEEE Transactions Software Engineering, ISSN: 0098-5589, (Impreso) 16(3), 360-369 (1990). http://www.computer.org/csdl/trans/ts/1990/03/e0360-abs.html Acceso: 2 de Abril (2013). 